\title{
THE INTENSITY PROFILE OF THE SOLAR SUPERGRANULATION
}

\author{
Nathan Goldbaum ${ }^{1}$, Mark P. Rast ${ }^{2,3}$, Ilaria Ermolli ${ }^{4}$, J. Summer Sands ${ }^{5}$, and Francesco Berrilli ${ }^{6}$ \\ ${ }^{1}$ Laboratory for Atmospheric and Space Physics, Department of Physics, University of Colorado, Boulder, CO 80309, USA \\ ${ }^{2}$ Laboratory for Atmospheric and Space Physics, Department of Astrophysical and Planetary Sciences, University of Colorado, Boulder, CO 80309, USA; \\ mark.rast@lasp.colorado.edu \\ ${ }^{3}$ High Altitude Observatory, National Center for Atmospheric Research ${ }^{7}$, P.O. Box 3000, Boulder, CO 80307, USA \\ ${ }^{4}$ INAF-Osservatorio Astronomico di Roma, Via di Frascati, 33, I-00040 Monte Porzio, Italy \\ ${ }^{5}$ SOARS and the High Altitude Observatory, National Center for Atmospheric Research ${ }^{7}$, P.O. Box 3000, Boulder, CO 80307, USA \\ ${ }^{6}$ Dipartimento di Fisica, Università di Roma Tor Vergata, Viale della Ricerca Scientifica, 1, I-00133 Roma, Italy \\ Received 2009 June 17; accepted 2009 September 28; published 2009 November 18
}

\begin{abstract}
We have measured the average radial (cell center to network boundary) profile of the continuum intensity contrast associated with supergranular flows using data from the Precision Solar Photometric Telescope at the Mauna Loa Solar Observatory. After removing the contribution of the network flux elements by the application of masks based on Ca II $\mathrm{K}$ intensity and averaging over more than $10^{5}$ supergranular cells, we find a $\sim 0.1 \%$ decrease in red and blue continuum intensity from the supergranular cell centers outward, corresponding to a $\sim 1.0 \mathrm{~K}$ decrease in brightness temperature across the cells. The radial intensity profile may be caused either by the thermal signal associated with the supergranular flows or a variation in the packing density of unresolved magnetic flux elements. These are not unambiguously distinguished by the observations, and we raise the possibility that the network magnetic fields play an active role in supergranular scale selection by enhancing the radiative cooling of the deep photosphere at the cell boundaries.
\end{abstract}

Key words: Sun: granulation - Sun: magnetic fields - Sun: photosphere

Online-only material: color figure

\section{INTRODUCTION}

The solar supergranulation was first observed by Hart (1954) as coherent banded velocity structures with length scales of approximately $75 \mathrm{Mm}$ in line-of-sight Doppler measurements at the Sun's limb. Subsequent work (Leighton et al. 1962; Simon \& Leighton 1964) concluded that the signal observed by Hart corresponds to a surface-filling cellular pattern of horizontally diverging flows with diameters of approximately $30 \mathrm{Mm}$, flow speeds of $\sim 500 \mathrm{~m} \mathrm{~s}^{-1}$, and lifetimes of $\sim 1$ day. The same studies reported the strong statistical correspondence between the chromospheric magnetic network observed in Ca II K images and the borders of the supergranular cells. This correlation was confirmed by Kueveler (1983).

Supergranulation is readily identified in time-averaged Doppler images (e.g., Hathaway et al. 2000), by tracking the motions of individual granules (e.g., Rieutord et al. 2008), or by using the correlation between the Ca II K network and the convergent flow boundaries (e.g., Berrilli et al. 1998). The characteristic cell sizes reported depend somewhat on the identification technique employed, ranging from $15 \mathrm{Mm}$ (DeRosa \& Toomre 2004) to $25 \mathrm{Mm}$ (Hirzberger et al. 2008) or $35 \mathrm{Mm}$ (Rieutord et al. 2008), and may vary with the solar cycle (Berrilli et al. 1999; Meunier et al. 2008). Supergranular flows are characterized by $\sim 0.07 \mathrm{~km} \mathrm{~s}^{-1}$ central upflows (Kueveler 1983) and well-observed horizontal outflows with average speeds of $\sim 0.5 \mathrm{~km} \mathrm{~s}^{-1}$ (Meunier et al. 2008) that converge on isolated sites of downflow at supergranular borders (Kueveler 1983). Measurements of downflow speeds are limited by the intrinsically small downflow velocities as well as the presence of the magnetic network fields (e.g., Frutiger \& Solanki 1998). Taken together, these observations suggest that fluid emerges near the supergranular cell center, flows outward toward the cell borders

\footnotetext{
7 NCAR is sponsored by the National Science Foundation.
}

advecting the magnetic field, before sinking back into the solar interior. It is thus generally inferred that supergranulation is thermally driven and has a convective origin.

Early on (Leighton et al. 1962), and later reinforced by the discovery of mesogranulation (November et al. 1981), it was suggested that the somewhat discrete scales of solar convection reflect the depths of hydrogen and helium ionization. This is not supported by detailed models of ionizing convection, which show that partial ionization is broadly distributed in depth (ionization/recombination occurs at very different depths in upflows and downflows) and causes both linear and finite amplitude destabilization of the flow, favoring high-speed smallscale motions (Rast 1991; Rast \& Toomre 1993; Rast 2001), not large-scale flows with length scales reflecting the poorly defined mean ionization depth. This has led to the suggestion that the more elusive mesogranular and the supergranular scales emerge directly from the self-organization of granular flows (e.g., Rieutord et al. 2000; Rast 2003a, and references therein). While unambiguously determining the driving mechanism from observations is likely difficult, measurement of the thermal perturbation associated with supergranular flows, the goal of our work, places constraints on these models.

Measurement of the radial (cell center to network boundary) profile of the supergranular intensity must overcome two challenges: the weak supergranular signal underlies intensity fluctuations due to both granulation and the magnetic network. The granular "noise" can be overcome by spatial and temporal averaging since granulation evolves on short timescales and small length scales compared to those of the supergranulation. The contribution due to the magnetic network, however, must be more carefully accounted for, since the network is itself closely associated with the supergranular flows. In the absence of a correction for the network contribution, the supergranular contrast at most continuum wavelengths peaks at the cell boundaries (Beckers 1968; Foukal \& Fowler 1984; Lin \& Kuhn 1992). 
Table 1

Supergranule Population Statistics

\begin{tabular}{ccccccc}
\hline \hline$\xi$ & $\left\langle r_{0}\right\rangle^{\mathrm{a}}$ & $\begin{array}{c}\sigma_{r_{0}} \\
(\mathrm{Mm})\end{array}$ & $\begin{array}{c}\langle A\rangle^{\mathrm{b}} \\
\left(\mathrm{Mm}^{2}\right)\end{array}$ & $\begin{array}{c}\sigma_{A} \\
\left(\mathrm{Mm}^{2}\right)\end{array}$ & $N^{\mathrm{c}}$ & $\begin{array}{c}\mathrm{S}^{\mathrm{d}} \\
(\mathrm{Mm})\end{array}$ \\
\hline-0.2 & 16.2 & 7.7 & 1202 & 1775 & 42374 & 7.3 \\
-0.4 & 14.3 & 5.1 & 776 & 675 & 111635 & 7.3 \\
-0.6 & 17.0 & 4.8 & 1004 & 592 & 179898 & 7.3 \\
-0.6 & 15.4 & 5.5 & 861 & 615 & 219773 & 2.2 \\
\hline
\end{tabular}

Notes.

a Average cell radius.

b Average cell area.

c Total number of cells identified.

${ }^{\mathrm{d}}$ Cell radius threshold.

This is seemingly inconsistent with models for the supergranulation in which warm fluid rises at the cell center and cool fluid descends into the solar interior at the network boundaries. However, these uncorrected contrast measurements are dominated by opacity changes due to the presence of magnetic flux elements (e.g., Spruit 1976; Pizzo et al. 1993; Steiner 2005; Criscuoli \& Rast 2009), obscuring any thermal effects due to temperature perturbations in the plasma. Once the magnetic and thermal contributions to the signal have been disentangled, a decrease in brightness from the center to the edge of an average supergranule has been reported (Rast 2003b; Meunier et al. 2007, 2008), corresponding to a center-to-edge temperature change of 0.8-2.8 K (Meunier et al. 2007, 2008). Our work confirms these earlier measurements with a higher degree of certainty and somewhat better spatial resolution, while employing identification and measurement methodologies distinctly different from those of Meunier and coworkers.

\section{ANALYSIS}

\subsection{The PSPT Data}

The Precision Solar Photometric Telescope (PSPT) is a $15 \mathrm{~cm}$ refracting telescope designed for high $(0.1 \%)$ pixelto-pixel photometric precision, operated by the High Altitude Observatory (HAO) at Mauna Loa Solar Observatory (MLSO). It produces $2048 \times 2048$ full-disk solar images $\left(\sim 1^{\prime \prime}\right.$ pixel $\left.^{-1}\right)$ with a $\sim 20$ minutes cadence, weather permitting, in five wavelength bands: red continuum (607.1 FWHM $0.5 \mathrm{~nm})$, blue continuum (409.4 FWHM 0.3 nm), CaII K (393.4 FWHM $0.3 \mathrm{~nm}$ ), and two narrowband Ca II $\mathrm{K}$ filters not used in this study. Data processing challenges and techniques are described in detail in Rast et al. (2008).

For this study, a total of 1051 image triplets, nearly simultaneous (interleaved during observation) red continuum, blue continuum, and Ca II K images, from the period 2005 January to 2008 March were selected. Selection was based on the quality of the red image (the images used in this study have maximum red and blue continuum limb widths of 2.5 and 3.5 pixels, respectively; see Rast et al. 2008) and the availability of images in all three wavelength bands. Since our final measurement of the average radial intensity profile of supergranular cells makes use of only a small fraction of the pixels in any given image, those remaining after masking out magnetic element contributions, the signal from many images must be combined into a statistically significant measurement. To this end, each image triplet is aligned, supergranules are identified in the central $512^{2}$ pixel region of all $1051 \mathrm{Ca}$ II $\mathrm{K}$ images, and the average intensity at each wavelength as a function of distance from the super- granular barycenter is computed, with a total of more than $10^{5}$ supergranules contributing to the final measurement.

\subsection{Data Analysis}

The network pattern associated with the supergranular flows is readily apparent by visual inspection of the PSPT Ca II K images, but unambiguous and automatic identification of this pattern is required to perform statistical analyses of a large ensemble of supergranules. Two identification methods are commonly reported in the literature. The first begins with local correlation tracking of granular features to determine the advective flow of the supergranulation (e.g., November \& Simon 1988; Potts \& Diver 2008). The divergence of the horizontal velocity field measured is then calculated and smoothed, and a steepest descent algorithm is applied to compute the cell boundaries (DeRosa \& Toomre 2004; Meunier et al. 2007). The second, the method employed here, makes use of the strong correlation between the borders of supergranules observed in velocity and the chromospheric network observed in the Ca II $\mathrm{K}$ band (Kueveler 1983), which in turn reflects the correlation between Ca II K emission and magnetic flux density (Skumanich et al. 1975; Schrijver et al. 1989; Harvey \& White 1999; Rast 2003b; Ortiz \& Rast 2005) and the convergent advection of magnetic elements into the supergranular boundaries. In this method, a computer vision algorithm, based on morphological operations that segment binarized Ca II K images (Berrilli et al. 1998, 1999, 2005), maps the connected network boundaries. Mixed methods are certainly feasible, and should be explored for consistency in future work.

We employ the iterative medial axis transform algorithm of Berrilli et al. (1998, 2005) to generate supergranular maps of the central $512^{2}$ portion of the PSPT Ca II K contrast images. ${ }^{8}$ The algorithm calculates a binary image from the Ca II K contrast image and uses this to perform a skeletonization. If $I_{K}(x, y)$ is the Ca II $\mathrm{K}$ contrast at pixel position $(x, y)$, then the binary image is that produced by a high pass threshold based on the local mean and standard deviation of the pixels in an $L \times L$ moving window, with the threshold function

$$
T(x, y)=\left\langle I_{K}(x, y)\right\rangle_{L}+\xi \sigma(x, y)_{L},
$$

where $\sigma(x, y)_{L}$ and $\left\langle I_{K}(x, y)\right\rangle_{L}$ are, respectively, the standard deviation and average intensity of the pixels in the $L \times L$ window centered at $(x, y) . \xi$ is a tunable parameter.

\footnotetext{
8 The contrast at each wavelength is defined as $I_{c} \equiv \frac{\delta I}{I_{0}}=\frac{I-I_{0}}{I_{0}}$, where $I$ is the observed gain-corrected intensity and $I_{0}$ is the measured center-to-limb function at that wavelength (Rast et al. 2008).
} 


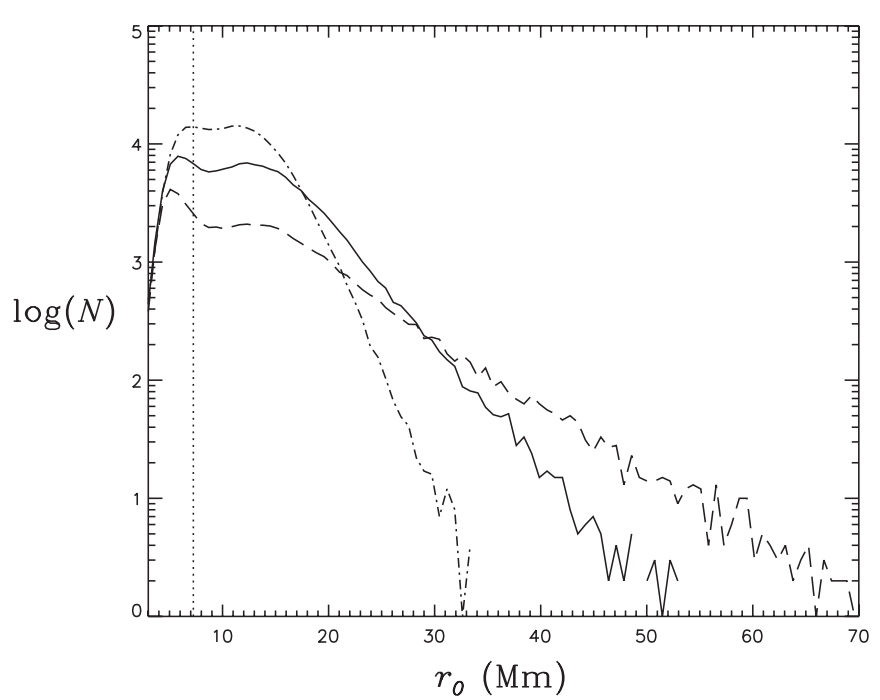

Figure 1. Logarithm of the number of supergranular cells identified in the images as a function of their size for three values of $\xi$. The dashed line corresponds to $\xi=-0.2$, the solid line corresponds to $\xi=-0.4$, and the dot-dashed line corresponds to $\xi=-0.6$. The dotted fiducial line indicates the length cutoff below which cells are not included in the annular averaging in the primary analysis.

For this study, we take $L=40$ pixels $(\sim 30 \mathrm{Mm})$, a typical supergranular length scale, and explore the sensitivity of the measured supergranualar properties to the value of $\xi$ (see Table 1). The parameter range examined follows that leading to optimal skeletonization (Berrilli et al. 1998, 2005). In general, $\xi$ is a negative number, and reasonable network-like structures are obtained with $0.0<|\xi|<1.0$. As $\xi$ approaches zero, the network cells produced by the algorithm tend to grow larger; as $\xi$ approaches unity, the network is fragmented and cell sizes decrease. This is apparent both visually and in the distribution of cell radii, $N\left(r_{0} ; \xi\right)$ (Figure 1$)$. Good, though never perfect, visual agreement between the identified cells and the Ca II K network apparent in the contrast images is obtained using $\xi \approx-0.4$ (see Figure 2). More importantly, the intensity profiles presented in Section 3 are robust over a significant range of $\xi$ values (Figure 3).

The skeletonization algorithm employed preserves connectivity within the image, making this algorithm ideal for segmenting the network pattern identified in images of moderate spatial resolution but also posing difficulties when that network breaks up into isolated points, as it does for PSPT Ca II K images of highest quality (those taken under the best seeing conditions at MLSO). To avoid these difficulties, we degrade all images before segmentation by convolution with a Gaussian so that the resulting limb width of the solar disk in the Ca II $\mathrm{K}$ image is 6.6 pixels. These smoothed images are used as inputs for the skeletonization procedure only. Once the supergranules have been identified, all further analysis is done at full resolution.

As discussed in Section 1, the measurement of the radial intensity profile of the supergranulation is complicated by the presence of the magnetic network. Network flux elements introduce opacity variations which contribute to positive continuum intensity fluctuations at the supergranular boundaries. The goal is to remove this contribution and examine pixels with only weak integrated magnetic flux density, in order to determine the intensity profile of the "unmagnetized" supergranular plasma. We note here, and discuss in Section 4, that this separation is likely never complete and is image resolution dependent. (a)

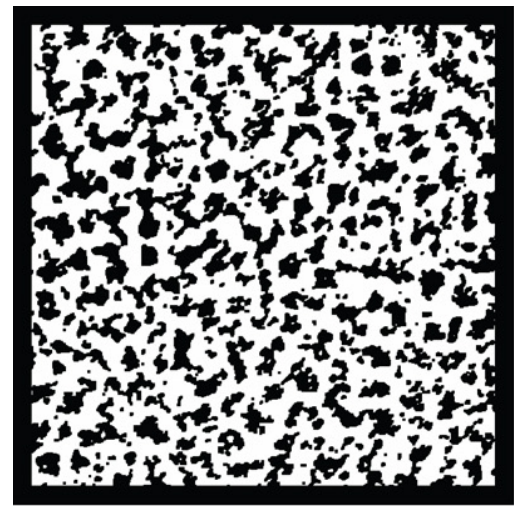

(b)

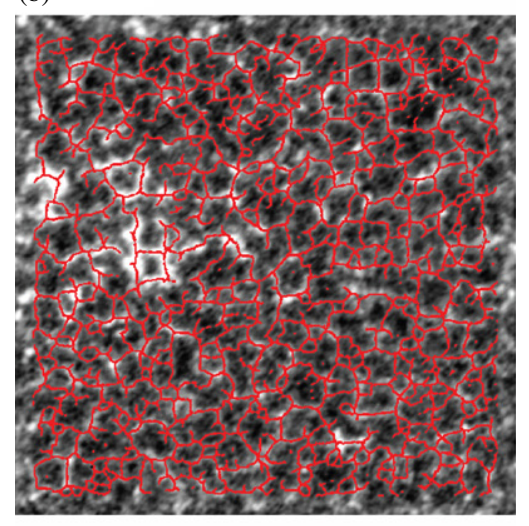

(c)

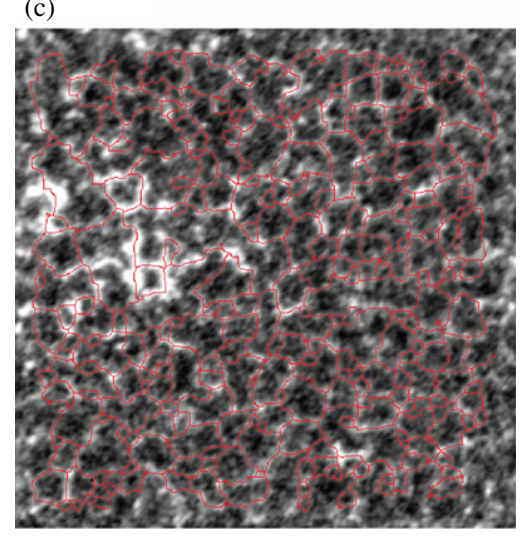

Figure 2. Three steps in the skeletonization process: the binary image (a) is skeletonized, yielding an initial approximation for the supergranular network. This initial skeleton is dilated (b) to include locally bright pixels that are connected to network. The resulting dilated network is then reskeletonized. Dialation and reskeletonization are repeated until differences between successive iterations become negligible (Berrilli et al. 2005). The resulting skeleton is closely aligned with the brightest intensity contours in the image. The image displayed was recorded at 18:50UT on 2005 March 7.

(A color version of this figure is available in the online journal.)

Meunier et al. (2007), employing both continuum images and magnetograms from the Solar and Heliospheric Observatory $(\mathrm{SOHO})$ Michelson Doppler Interferometer (MDI), evaluate several possible strategies for removing or accounting for the magnetic element contribution to the intensity signal. Intensity correction, based on a measured magnetic-flux continuumintensity relation, proved inadequate, likely because this relationship has significant scatter due to the unresolved magnetic substructure of the elements (Criscuoli \& Rast 2009). Direct masking (Rast 2003b; Rast et al. 2008) proves more useful, and 


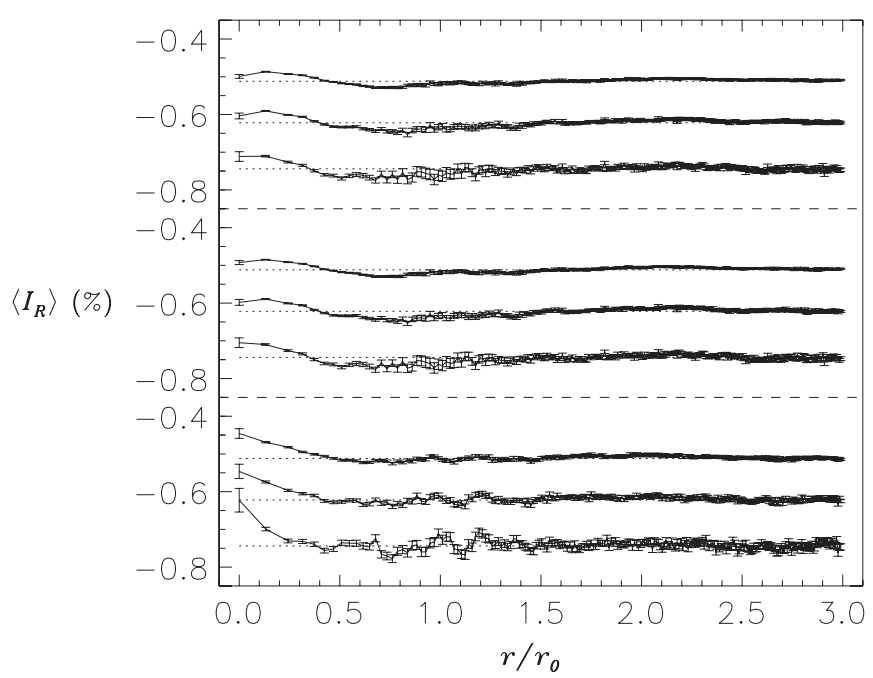

Figure 3. Average supergranular contrast profiles $I_{R}\left(r / r_{0}\right)$ for masks M5, M6, and $\mathrm{M} 7$ and identification parameters, $\xi=-0.6$ with cells smaller than $7.25 \mathrm{Mm}$ excluded (top), $\xi=-0.6$ with cells smaller than $2.2 \mathrm{Mm}$ excluded (middle), and $\xi=-0.2$ with cells smaller than $7.25 \mathrm{Mm}$ excluded (bottom). Dotted fiducial lines indicate the average intensity of the unmasked pixels in the images.

after image alignment and resizing, we employ that technique here. The image masks are based on both red continuum and $\mathrm{Ca}$ II $\mathrm{K}$ intensity which serve as a proxy for the magnetic flux density. The red continuum image is used to eliminate dark pore, umbral, and penumbral pixels from further consideration. Bright $\mathrm{Ca}$ II $\mathrm{K}$ pixels are eliminated with a series of increasingly severe contrast thresholds. As summarized in Table 3 of Rast et al. (2008), these masks together eliminate from $0.1 \%$ to $99.96 \%$ of the pixels in a given image from consideration, depending on the severity of the Ca II K threshold.

Continuum intensity fluctuations in the solar photosphere are dominated by granulation. Since the lifetime of a supergranule exceeds that of a granule by a factor of about 100, the intensity fluctuations of individual supergranules should be marginally detectable (equal in magnitude to those of granules) by observations on a subgranular-lifetime cadence if their amplitude is about 10 times smaller than those of granulation. Unresolved quiet-Sun granulation displays an average red continuum contrast of $\sim 1 \%$ in PSPT images, making supergranular contrasts of $\sim 0.1 \%$ just marginally detectable by image averaging over a supergranular lifetime. While, as will be shown in Section 3, the nonmagnetic supergranular signal is of this order, continuous high-quality observation over a supergranular lifetime, is not possible from the ground. This motivates measurements that average over many supergranules, sacrificing knowledge of individual cell profiles for vastly increased signal to noise.

Using the supergranular maps and masks described, we measured the azimuthally averaged supergranular radial intensity profile in the red, blue, and Ca II K contrast images. The mean contrast was determined for each of 225 equal-area annuli extending outward from the barycenter of the supergranules to $3.0 r_{0}$, where $r_{0}$ is average radius of the individual supergranule measured in the four grid-aligned directions. With this scaling, the outer radius of the 25th annulus corresponds to the measured cell's average radius. To avoid nested averages, the contrast values for all of the pixels in any particular annulus were tabulated for all supergranules, and the mean and standard deviation of the contrast in each radial bin were calculated over the entire data set. The central contrast value for each supergranule was also recorded, and since its average combines only single pixel values from the individual supergranules, the random error in the final profile at $r=0$ is larger than at larger radii.

We note, that by averaging intensities using an annular sampling, we are implicitly assuming that supergranules are circular. Departures from circularity, evident for most supergranules under consideration, smears the resulting profile, and our final measurement thus represents a lower limit to the contrast variation across a supergranular cell. Moreover, while equal-area annuli allow measurements of equal significance at all radii for any single supergranule, the measurements have greater variance for small supergranules than large, since the number of radii is fixed and thus the number of pixels in each annulus goes down with supergranular size. We ameliorate this to some degree by discarding small cells (average radii less than 10 pixels, $\sim 7.3 \mathrm{Mm}$ ) as too poorly sampled, and the results are qualitatively insensitive to the exact threshold (Figure 3 ). The discarded small cells are about a factor of 2 smaller than the average supergranule in the images, and belong to the lower portion of a somewhat bimodal size distribution of radii (Figure 1). They appear to largely represent substructure (perhaps mesogranulation) within supergranular cells.

The measurements described were made for all the supergranular cells identified in the images (but not discarded as too small) after application of the eight Ca II K masks, with only unmasked pixels contributing to the analysis. Since the pixels with the lowest magnetic field strength have on average the lowest contrast, the mean intensity at all radii decreases as the applied mask becomes increasingly severe (Figure 4). Of more interest is the radial variation in the contrast profile (cell center to network boundary). To confirm that variation for each mask value is a property of the supergranular cells and not due to unforeseen systematic error, the averaging procedure described was also performed using random pixel locations as cell centers. Flat profiles, corresponding to the mean intensity of the images after masking (dotted lines in Figure 4), were always found for such random sampling.

\section{RESULTS}

The main results of this study are shown in Figure 4 as plots of the average contrast $\left\langle I_{c}\right\rangle$ at each wavelength as a function of radial position $r / r_{0}$. The results after increasingly severe masking (top to bottom) are presented as separate curves. In Ca II K (Figure 4(a)), the strong increase in brightness associated with the magnetic network in the absence of masking (top most curve) is seen as a $\sim 5 \%$ increase in average contrast at $r / r_{o}=1$ above that at cell centers. The curve approaches the mean value of the image at distances which exceed the correlation length of the network, at which point annular sampling about the cell centers and complete sampling are equivalent. Notably, the three most severe masks (99.96\% of the brightest pixels are eliminated by the most severe) applied to Ca II $\mathrm{K}$ images produce profiles with a residual contrast difference of only $\sim 0.05 \%-0.2 \%$ between cell centers and boundaries, indicating the elimination of nearly all magnetic element contributions, but that residual has the same sense as the unmasked profile, dimmer at cell center and brighter near the boundaries between cells.

The red and blue continuum images (Figures 4(b) and (c)) show similar network brightening when no masking is applied, with a $\sim 0.3 \%-0.4 \%$ increase in average contrast from the cell centers to the boundaries, consistent with previous measurements. This magnetic element contribution to the intensity gradually diminishes as the severity of masking increases. After application of the more severe masks, the plots show a slight 

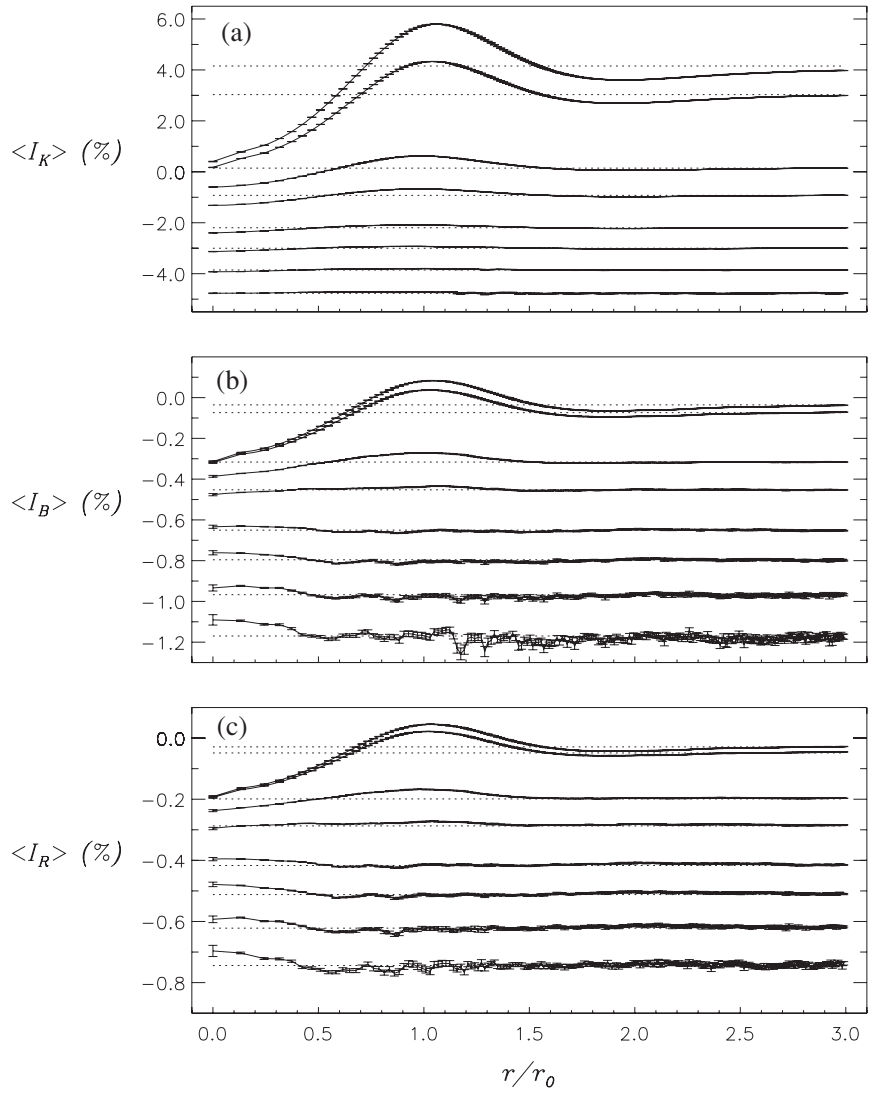

Figure 4. Average supergranular contrast profiles $I_{c}\left(r / r_{0}\right)$ in the (a) Ca II $\mathrm{K}$, (b) blue continuum, and (c) red continuum wavelength bands for each value of the Ca II $\mathrm{K}$ masking threshold applied. Dotted fiducial lines indicate the average intensity of the unmasked pixels in the images. Profiles found using the least severe mask (M0) are at top of each plot, with each curve below found using progressively more severe activity masks (mask M7 used for the bottommost profile; see Table 3 in Rast et al. 2008 for mask definitions). The peak at $r / r_{0}=1$ for weak masking reflects the contribution from magnetic network. With increasingly severe masking the enhanced intensity at the cell boundaries decreases, eventually dropping, at continuum wavelengths for the most severe mask values, below the intensity observed at the cell centers. These profiles are for cells identified using the identification parameter $\xi=-0.4$ (Row 2, Table 1) and a minimum cell radius of 10 pixels $(\sim 7.3 \mathrm{Mm})$.

decrease in the average supergranular contrast toward the cell boundaries. Notably, this is true even though the opposite trend continues to be measured in Ca II $\mathrm{K}$ for the same mask values (above). Moreover, there is some weak indication that, not only does the contrast decrease with distance from the supergranular center, but the cell boundaries are on average slightly darker than the image mean.

The decrease in contrast from the supergranular cell centers outward after masking the magnetic element contribution is found at both continuum wavelengths, and has a maximum amplitude of $\sim 0.05 \%-0.1 \%$. The differences between the amplitudes of the signal at the two wavelengths is consistent with a single brightness temperature perturbation (below). Since more than $10^{5}$ supergranules are included in the measurement, random errors (or fluctuations, since they are largely solar in origin) are small (the $1 \sigma$ range is over plotted in Figure 4) and the statistical significance of the measurement is high. The results are fairly insensitive to the number of annuli employed. Increasing the number not only increases the radial resolution, but also the random/granular noise. Moreover, some inherent smoothing of the profile occurs, independent of the number of anulli employed, because the geometry of the supergranules

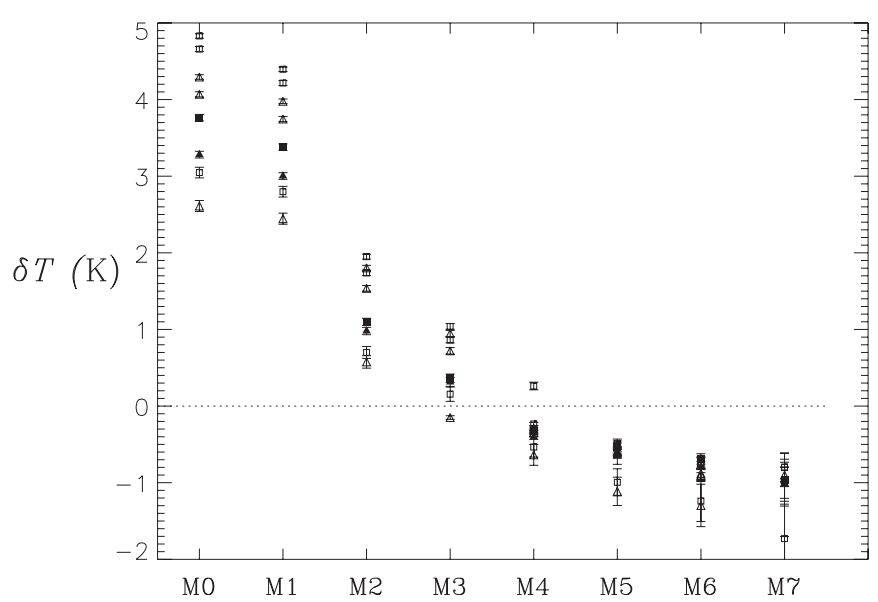

Figure 5. Solid symbols plot the red continuum (triangles) and blue continuum (squares) brightness temperature difference across the supergranular cells as derived from the maxima and minima of the intensity profiles of Figure 4 between $r / r_{0}=0$ and 1 . The dotted fiducial line indicates $\delta T=0$. With no masking, we find an average temperature increase of 3-4 K from center to edge across the supergranular cells. For more severe masking (M4 through M7), the brightness temperature difference (edge minus center) becomes negative, apparently converging on a value near $-1 \mathrm{~K}$. Open symbols plot the same quantities after varying the identification scheme parameters (see the text).

is not circular as assumed. Tests with random pixel locations (Section 2) suggest that aside from this smoothing, systematic errors in the measurement are even smaller than those due to random fluctuations. The results found here using $>10^{5}$ supergranules thus confirm with much greater confidence those of Rast (2003b), who tentatively detected the signal using the same techniques applied to 7300 supergranules, and Meunier et al. (2007) who confidently detected such a signature using quite different methodologies on 7629 supergranules.

Assuming that the observed intensities at the continuum wavelengths are due to small temperature perturbations of a thermal blackbody, the supergranular center-to-edge contrast measured can be converted for each mask value into a brightness temperature difference. These are plotted for the red and blue continuum images with filled symbols in Figure 5, where the brightness temperatures were found using the observed maximum and minimum contrast values of the profiles between $r / r_{0}=0$ and 1 (Figure 4). With no masking, the temperature fluctuations associated with the bright magnetic network have amplitudes of $\sim 3-4 \mathrm{~K}$. With increasingly severe masking, the fluctuations become negative, with a value near $-1 \mathrm{~K}$ for the most severe masks. On average, the brightness temperature of the "nonmagnetized" supergranular plasma is $\sim 1 \mathrm{~K}$ greater at the supergranule center than the edge, consistent with the range $0.8-2.8 \mathrm{~K}$ cited by Meunier et al. $(2007,2008)$.

These results are only weakly sensitive to variation of the identification-scheme parameters. Contrast profiles resulting from identical analysis of cells identified using three choices of $\xi$ and cutoff size threshold are plotted in Figure 3. These curves, for the three most severe masks, are qualitatively and quantitatively similar to those found using $\xi=-0.4$ and a cutoff of 10 pixels (Figure 4). The corresponding average brightness temperature contrast across the cells is plotted as open symbols in Figure 5. The results are all generally within $2 \mathrm{~K}$, with the plot scatter reflecting the sensitivity of the maximum and minimum profile intensities to the identification-scheme parameters, and the error bars indicating the formal propagation of random error (largely granular fluctuations) in the intensity measurement. 


\section{CONCLUSION}

In this work, we have successfully measured the mean supergranular radial intensity profile. We have employed maps and masks based on the $\mathrm{Ca}$ II $\mathrm{K}$ intensity of the magnetic network. After application of the most severe masks, we have found, with a high degree of confidence, that the centers of supergranules are on average $\sim 0.1 \%$ brighter than their borders. This corresponds to a brightness temperature difference of $\sim 1 \mathrm{~K}$. The detection, near the limit of the PSPT's capabilities, is consistent with previous measurements (Rast 2003b; Meunier et al. 2007, 2008), this despite the fact that we employ very different methodologies from Meunier et al. for detection, mapping, and masking of the supergranular cells.

Is the amplitude of the signal observed consistent with a convective origin for the supergranulation? A simple balancing of the kinetic energy of the motions and buoyancy work over the length of parcel travel (Frazier 1970),

$$
\frac{1}{2} \rho v^{2} \approx \rho^{\prime} \frac{l}{2} \epsilon
$$

where $\rho$ is the plasma density, $v$ is its velocity, $g$ is the acceleration due to gravity, $l$ is the length of parcel travel, and $\epsilon$ is a measure of convective efficiency, yields, when evaluated for the linear displacement of an ideal gas parcel in pressure equilibrium, expected temperature perturbations:

$$
T^{\prime} \approx \frac{T v^{2}}{g l \epsilon},
$$

where $T$ is the plasma temperature. For granulation, $T^{\prime}$ expected from a strict balance of kinetic energy and buoyancy work (taking $\epsilon=1$ ) is $33 \mathrm{~K}$ and its measured value in the photosphere is $\sim 300 \mathrm{~K}$, suggesting that $\epsilon=0.1$. Numerical models of granular flow suggest that the actual continuum intensity contrast of granulation may in fact be a factor of 2 yet higher (Danilovic et al. 2008; Nordlund et al. 2009). The same value for $\epsilon$ is deduced for supergranulation, taking $v \sim 0.2 \mathrm{~km} \mathrm{~s}^{-1}$, $T \sim 10^{4} \mathrm{~K}, g \sim 0.3 \mathrm{~km} \mathrm{~s}^{-2}, l \sim 15 \mathrm{Mm}$, and the observed temperature contrast measured here $T^{\prime} \sim 1 \mathrm{~K}$. So while the thermal signal measured is small, both granulation and supergranulation show continuum intensity contrasts about an order of magnitude larger than that expected from the highly simplified parcel argument above but significantly smaller than one might expect from numerical models of granular flows.

A convective origin for the supergranulation may not be an exclusive explanation for the measured intensity perturbations. We note that the masking procedure employed removes bright magnetic flux elements only in so far as they can be resolved. Variation in packing density of unresolved magnetic flux elements may contribute to the brightness profiles measured. Rather than reflecting a thermal perturbation, the positive continuum intensity signal measured may instead indicate that on average the central regions of supergranular cells contain many small-scale unresolved flux elements that subsequently coalesce as they are advected toward the boundary rendering them resolvable to our masking procedure. This interpretation is suggested by the apparent ubiquitous presence of inter-network flux elements (e.g., de Wijn et al. 2008, and references therein), and the degree of masking required to remove the magnetic element signature from the measurement. Average profiles with enhanced cell center intensities are observed at the red and blue continuum wavelengths only after more than $95 \%$ of the pixels have been masked, leaving only pixels with magnetic flux density $\lesssim 0.7 \mathrm{G}$ (see Table 3 in Rast et al. 2008 for tabulated properties of the eight masks employed). The Ca II K intensity, on the other hand, remains enhanced at network cell boundaries even after more severe masking (dark cell centers and brighter boundaries are still seen in Ca II K after masking $99.96 \%$ of the brightest pixels, down to average magnetic flux densities of $\sim 0.4 \mathrm{G})$. It thus suggests that the number density of unmasked flux elements is less at the cell centers than the network boundary for all mask values. Three possibilities remain to reconcile these profiles. (1) The trend in magnetic flux density suggested by the masking sequence on $\mathrm{Ca}$ II $\mathrm{K}$ images does not continue at unresolved scales and the continuum signal reflects the presence of greater amounts of unresolved flux at cell centers than at the cell boundaries. (2) An underlying thermal perturbation dominates the contribution of residual flux elements to the continuum, but not the $\mathrm{Ca}$ II $\mathrm{K}$, intensities after severe masking (i.e., we have indeed uncovered the convective signature of supergranulation). (3) The radiative properties of very weak magnetic flux elements or weak field regions, if the field is no longer collected into semidiscrete bundles at those scales, are such that unlike at greater flux densities, their contrast is positive in $\mathrm{Ca}$ II $\mathrm{K}$ but negative at both the red and blue continuum wavelengths. Without detailed modeling of the flow, flux distribution, and radiation field it may not be possible to unambiguously untangle these possibilities.

Finally, we note that the severe masking required to uncover a possible thermal signature of the supergranulation suggests that the concept of "quiet-Sun" may be inappropriate. Opacity variations due to the presence of a magnetic field may be important in determining intensity fluctuations on the Sun even at very low field strengths and over a range of spatial and temporal scales. The presence of magnetic network may play an important active role in the driving of the supergranular flow by radiatively cooling the deeper layers of the photosphere (e.g., Criscuoli \& Rast 2009). This would imply that the network field is not strictly a passive tracer of the supergranular flow, but rather may play an active dynamic role in supergranular scale selection by inducing radiative perturbations at depth where the density and pressure scale heights are greater. This may provide an explanation for why strictly hydrodynamic simulations have difficulty unambiguously obtaining supergranular scales (Ustyugov 2008; Stein et al. 2009), though domain sizes are also currently still quite restrictive.

Special thanks to S. Criscuoli, J. Harder, and an anonymous referee.

\section{REFERENCES}

Beckers, J. M. 1968, Sol. Phys., 5, 309

Berrilli, F., Ermolli, I., Florio, A., \& Pietropaolo, E. 1999, A\&A, 344, 965

Berrilli, F., Florio, A., \& Ermolli, I. 1998, Sol. Phys., 180, 29

Berrilli, F., Moro, D. D., Florio, A., \& Santillo, L. 2005, Sol. Phys., 228, 81

Criscuoli, S., \& Rast, M. P. 2009, A\&A, 495, 621

Danilovic, S., Gandorfer, A., Lagg, A., Schüssler, M., Solanki, S. K., Vögler, A., Katsukawa, Y., \& Tsuneta, S. 2008, A\&A, 484, L17

DeRosa, M. L., \& Toomre, J. 2004, ApJ, 616, 1242

de Wijn, A. G., Lite, B. W., Berger, T. E., Frank, Z. A., Tarbell, T. D., \& Ishikawa, R. 2008, ApJ, 684, 1469

Foukal, P., \& Fowler, L. 1984, ApJ, 281, 442

Frazier, E. N. 1970, Sol. Phys., 14, 89

Frutiger, C., \& Solanki, S. K. 1998, A\&A, 336, L65

Hart, A. B. 1954, MNRAS, 114, 17

Harvey, K. L., \& Wite, O. R. 1999, ApJ, 515, 812

Hathaway, D. H., Beck, J. G., Bogart, R. S., Bachmann, K. T., Khatri, G., Petitto, J. M., Han, S., \& Raymond, J. 2000, Sol. Phys., 193, 299 
Hirzberger, J., Gizon, L., Solanki, S. K., \& Duvall, T. L. 2008, Sol. Phys., 251, 417

Kueveler, G. 1983, Sol. Phys., 88, 13

Leighton, R. B., Noyes, R. W., \& Simon, G. W. 1962, ApJ, 135, 474

Lin, H., \& Kuhn, J. R. 1992, Sol. Phys., 141, 1

Meunier, N., Roudier, T., \& Rieutord, M. 2008, A\&A, 488, 1109

Meunier, N., Tkaczuk, R., \& Roudier, T. 2007, A\&A, 463, 745

Nordlund, A., Stein, R. F., \& Asplund, M. 2009, Living Rev. Sol. Phys., 6, 2 (http://solarphysics.livingreviews.org/Articles/lrsp-2009-2/ (cited on 9/9/09))

November, L. J., \& Simon, G. W. 1988, ApJ, 333, 427

November, L. J., Toomre, J., Gebbie, K. B., \& Simon, G. W. 1981, ApJ, 245 , L123

Ortiz, A., \& Rast, M. 2005, Mem. Soc. Astron. Ital., 76, 1018

Pizzo, V. J., MacGregor, K. B., \& Kunasz, P. B. 1993, ApJ, 413, 764

Potts, H. E., \& Diver, D. A. 2008, Sol. Phys., 248, 263

Rast, M. P. 1991, in Lecture Notes in Physics, Vol. 388, Challenges to Theories of the Structure of Moderate-Mass Stars, ed. D. Gough \& J. Toomre (Berlin: Springer), 179

Rast, M. P. 2001, ApJ, 561, L191
Rast, M. P. 2003a, ApJ, 597, 1200

Rast, M. P. 2003b, in Proc. SOHO 12/GONG + 2002, Vol. 517, Local and Global Helioseismology: The Present and Future, ed. H. Sawaya-Lacoste (Noordwijk: ESA), 163

Rast, M. P., Ortiz, A., \& Meisner, R. W. 2008, ApJ, 673, 1209

Rast, M. P., \& Toomre, J. 1993, ApJ, 419, 224

Rieutord, M., Meunier, N., Roudier, T., Rondi, S., Beigbeder, F., \& Parès, L. 2008, A\&A, 479, L17

Rieutord, M., Roudier, T., Malherbe, J. M., \& Rincon, F. 2000, A\&A, 357, 1063

Schrijver, C. J., Cote, J., Zwaan, C., \& Saar, S. H. 1989, ApJ, 337, 964

Simon, G. W., \& Leighton, R. B. 1964, ApJ, 140, 1120

Skumanich, A., Smythe, C., \& Frazier, E. N. 1975, ApJ, 200, 747

Spruit, H. C. 1976, Sol. Phys., 50, 269

Stein, R. F., Georgobiani, D., Schafenberger, W., Nordlund, Å., \& Benson, D. 2009, in Proc. 15th Cambridge Workshop, Vol. 1094, Cool Stars, Stellar Systems, and the Sun, ed. E. Stempels (Melville, NY: AIP), 764

Steiner, O. 2005, A\&A, 430, 691

Ustyugov, S. D. 2008, in AIP Conf. Proc. 1043, Exploring the Solar System and the Universe, ed. V. Mioc, C. Dumitrache, \& N. A. Popescu (Melville, NY: AIP), 234 